\title{
From Playing to Programming: The Effect of Video Game Play on Confidence with Computers and an Interest in Computer Science
}

\author{
by Rebecca Sevin and Whitney DeCamp \\ Western Michigan University; Western Michigan University
}

Sociological Research Online, 21 (3), 16

$<$ http://www.socresonline.org.uk/21/3/16.html>

DOI: $10.5153 /$ sro.4082

Received: 25 Nov 2015 | Accepted: 22 Aug 2016 I Published: 31 Aug 2016

\begin{abstract}
Research on the effects of playing video games has been limited by a preoccupation with possible negative repercussions. Nevertheless, research has shown that video games can have positive effects on young players' social lives. The existing body of research, however, has largely ignored the more computer-related aspects of video game play and its effects. This study provides empirical evidence to support theoretical arguments about the relationship between playing video games and computers. The type of scientific thinking encouraged by video games and the technological abilities needed to play video games is suggested to result in an increase in players' confidence with computers and interest in computer science. These potential relationships are examined using data from over 1,000 undergraduate students to empirically assess the relationship between video game play and: 1) confidence with computers, and 2) interest in computer science. The results indicate that game play is statistically significant as a predictor of confidence and interest. In comparison to the other predictors in the model, the strength of the effect from playing video games is relatively very strong. The findings suggest that exposure to video games as a recreational technology help inform players' abilities with nonrecreational technology and build an interest in technology in general.
\end{abstract}

Keywords: Sociology, Video Games, Computer Science, Survey, Leisure.

\section{Introduction}

Too often, the benefits of leisure activity have been severely underestimated. We consider only the conventional benefit of relaxing and distancing ourselves from life stressors, but the benefits of leisure activities far surpass being only a simple distraction. Based on the chosen activity, participants can gain a variety of other benefits that reach across multiple aspects of their lives. One particular benefit is that leisure activities can positively shape specific aspects of our self-perceptions. For example, women participating in belly dance reported feeling empowered and more comfortable with their bodies as a result of their participation, even despite past physical victimization (Moe 2014). Others who participate in textile crafting as leisure activity have developed their values of sustainability through crafting by becoming more aware of their consumption and waste (Pöllänen 2015). Throughout the literature leisure has been shown to affirm pre-existing identities, but it is also a place for forming identities and personal growth (Mannell 2007). This development of the self through leisure is particularly important for activities that are often aimed at younger audiences, such as video games. The possible negative influences that video games can have on the development of the self have been heavily scrutinized in the past, but the ways in which interacting with video games may have a positive influence, particularly on younger players, have been largely overlooked. discussion around video games in recent years has focused primarily on the possibility of them inspiring violence in players and on other concerns about negative side effects (e.g., addiction). Indeed, these debates continue unabated (e.g., Bushman \& Pollard-Sacks 2014; Ferguson et al.2014; Hoffman 2014; Gentile et al. 2011). Although it would be premature to dismiss the potential negative outcomes at this time, the almost exclusive focus on negative outcomes and inattention to potential positive outcomes has been a barrier to understanding the benefits that may come with playing video games. In addition, the emphasis on violence has resulted in research that focuses predominately on first person shooter (FPS) video games. This ignores the fact that there is a wide array of video game genres, and it supports the ethnocentric idea that only FPS games (primarily American-produced games) matter and excludes the large number of games imported from Japan and other countries, in addition to the larger variety created in America as well (ESA 2014). The present study seeks to address this oversight in the research by examining possible social benefits of playing video games - namely increased confidence with computers and interest in computer science - from playing games from a multitude of different genres. 
Over the past two decades, much of the research published on video games has focused on possible negative outcomes, particularly with regards to violence. Using a variety of measurements and analytic techniques, research in this area has reported both evidence of a connection between violent games and violence or aggressive behavior (e.g., Anderson et al. 2008; Bartholow et al. 2005; Carnagey et al. 2007) and a lack of such an effect (e.g., Adachi \& Willoughby 2011; DeCamp 2015; Ferguson et al. 2008; Gunter \& Daly 2012). In short, evidence about the connection is mixed at best. However, violence and aggression make up only a couple of the myriad of possible outcomes from gaming. Moreover, violent games, such as FPS games, make up the minority of games available to consumers. Even in this limited pool of games, FPS games require players to adeptly navigate a 3-dimensional space and take in audio and visual information quickly. This has been empirically shown to result in better spatial reasoning skills (Cherney 2008; De Lisi \& Wolford, 2002; Feng et al. 2007; Green \& Bavelier 2007), multisensory processing (Donohue et al. 2010), and visual working memory (Blacker et al. 2014). Although no other genre of games has received as much attention as FPS games, a limited number of studies have examined the effects of other genres on players. Massively multiplayer online games (MMOs) have received some attention for the social aspects of the game and one study suggests that MMOs can develop leadership skills, such as fast-paced decision-making and sharing/delegating authority with others (Lisk et al. 2012). Other studies have explored the potential of educational video games for being a better means of teaching students than traditional lecture method (Mayo 2009).

There is also a growing body of video game research focused more on the social effects of gaming. An early study on the effects of video games on children's leisure activities, schoolwork, and peer involvement found that school work and peer activities were largely unaffected by whether a child owned a game console or not (Creasey \& Myers 1986). Other studies have suggested part of the appeal of video games for boys is talking about them at school, suggesting that even playing alone is meant to serve a social function (Kunter, \& Olson 2008). One study examining the mood management abilities of video games found that violent video games reduced depression and hostile feelings in young adults (Ferguson \& Rueda 2010). Others researchers have suggested that video games may make players more likely to be helpful to others (Rosenberg et al. 2013), and plot-heavy games encourage children to read books based on video games (Jolly 2008). There is also a growing body of research regarding the role that video games and video game logic could play in education (Young et al. 2012). Despite the new and expanding area of research into the positive effects of gaming, however, very few empirical studies have examined the relationship between video games and computer science.

\section{Video Games and Computers}

As previously noted, very few studies have examined the connection between playing video games and having an interest in computer science. Those that have examined the effect tend to find support for such a hypothesis. One researcher, for example, observed that when teaching high school students how to use 3dimensional software in an art course, experiences with video games were informing how the students interacted with the program and made artistic choices (Gill 2009). Moreover, a study of African American high school students working as video game testers found that $65 \%$ of the participants studied computer science after high school (DiSalvo et al. 2014). Although the study focused on students who were paid to test video games, which already represents a biased sample, the evidence nonetheless supports the notion that video games could be used to create an interest in computer science.

In addition to the more specific benefits outlined in past studies, it has also been theorized that the logic of video games encourages a particular way of thinking and interpreting information in players. In What Video Games Have to Teach Us about Learning and Literacy (2003), James Paul Gee argues that the logic used when playing video games is similar to the logic behind the scientific method. He suggests that video games teach players to identify patterns, form a hypothesis, test this hypothesis, and then evaluate the results. Gee goes as far as to point out that science classes often give children information about the world in the form of facts, but that video games give children a new world where they can discover things on their own. In the classroom, students are taught the scientific method, but in video games they can actually employ it.

Another author has extended this argument by suggesting that video games help prepare players for the science, technology, engineering, and mathematics (STEM) fields by teaching them to think and reason in a scientific manner (Johnson 2005). It could also be argued that the more types of video games a player is exposed to, the more practice they will have using this type of thinking. Because gameplay is often similar in games of the same genre, once a player has identified certain patterns in one game, they can apply this knowledge to the next game. This is part of why experienced video game players can learn and master a new video game much faster than inexperienced players. Playing different games and different genres of games requires constantly finding new patterns and forming new hypotheses. Empirical research has found significant effects between playing video games and problem solving skills. A longitudinal study by Adachi and Willoughby (2013) found that players of strategic video games had higher self-reported problem solving skills and also had higher academic achievement via an indirect effect. The study is also unique in its focus on strategy games over the more dominant focus of FPS games that currently exists in the literature.

When taken together, the past literature suggests that video games may increase a player's technological literacy. The definitions of technological literacy vary, but in general it refers to an individual's ability to use technology effectively in solving problems. More specifically it is has been argued that interactions and experience with video games can address aspects of each of three dimensions: knowledge, critical thinking or decision making, and capability (Gamire \& Pearson 2006). Within games, players are encouraged to test boundaries (Gee 2003) which gives players a knowledge of constraints and limitations. The dimension of critical thinking and decision making, which video games are argued to develop in players, are similar to that of the 
scientific reasoning (Johnson 2005). Finally, capabilities refers to what we often more generally think of as technological skills. A person's experiences with technology and ability to identify and troubleshoot problems. Setting up video games and dealing with any problems that arise with the physical gaming hardware is one of example of this, but even more so is that many video games require a player to negotiate 3-D space quickly and effectively. These examples apply to even casual video game players, but those more invested in video games are even more likely to have experience understanding the engines used to create different types of games or nuances in hardware between different console systems. Unlike many other forms of technology that serve primarily utilitarian purposes, video games are first and foremost a recreational technology. The vast majority of players are unlikely to engage with video games with the purpose of developing technological literacy, but in the pursuit of recreation end up developing such literacy nonetheless. This applies even if they only engage in the most casual sense, but especially for players more intensely engaged with gaming.

The current study builds on these arguments by exploring the relationship between playing a variety of video games and confidence with computers, as well as interest in computer science. Because video games are a leisure activity often aimed at younger audiences, players may develop a familiarity with digital technology that can later be examined as college students. Through video games players engage with a number of different interfaces, patterns, logic, and potential situations for troubleshooting technical issues. Because of this experience, the more players engage with video games, the more likely it is that they will become interested in other functional aspects of digital technology, namely computers, even if they do not use computers as their primary gaming device. However, the argument is not that playing Minesweeperfor several hours each day will result in the development of technological literacy. Instead, it is the breadth of experience with video games that leads to stronger technological literacy. Each genre of game offers a different mechanical emphasis (thought this can vary greatly even within a genre). For example, FPS games such as Halo series require players to navigate 3-D space adeptly. Strategy games like the Civilizations series require plays to manage a great deal of data management, both visually through the game's graphics, but also through navigating multiple tables of data. The more advanced players often seek the most efficient means to manage these data. Even Massively Multiplayer Online games (MMOs), such as World of Warcarft, require players to find ways to communicate effectively with each other to accomplish goals, either by text-based or voice-based chats. With each genre a player engages in, they have the opportunity to develop skills related to other areas of technology if they want to master the game. Through multiple means - such as scores, the ability to advance thorough a game's story, or achieving higher online rankings - these skills are developed in a setting that is fun and rewarding, leading to a positive association with these skills and tasks. The result is that a breadth of experience with video games potentially results in more confidence in abilities with a computer and an interest in computer science. The present study investigates this potential relationship.

\section{Methods}

3.1 Data were collected from full-time undergraduate students at a large, public, American Midwestern university during the spring semester of 2014. The university provided the researchers with a list of 6,000 randomly selected full-time undergraduate students. These students were sent an invitation via email to participate in a web survey and were promised anonymity by not having any identifying information included in the final dataset. There was no incentive offered for participating. After sending the invitation and a few reminder emails, 1,124 individuals participated.[1] The sample is $51 \%$ female and $78 \%$ non-Hispanic white, which are proportions within a few percentage points of the population in the region of the United States from which the sample was drawn. The median age of the sample is 20 years old, making it typical for college student age ranges.

\section{Dependent Variables}

From a variety of indicators about attitudes towards computers, two scales were constructed: confidence with computers and interest in computer science. Various statements were listed on the survey and participants were asked to select one of seven possible responses, ranging from strongly disagree to strongly agree, for each statement. The indicators used to construct confidence with computers ask about situations (e.g., "I often help my parents with computers") as well as their general attitude towards computers (e.g., "I believe it's worth my time to just play around on computers") to form a comprehensive impression of the subject's relationship with computers and particularly with troubleshooting computers. The full list of statements used is included as an appendix. The measures were combined by using a participant's mean score across all indicators. A reliability analysis indicated that these indicators scaled together with very high reliability $(a=.909)$. Descriptive statistics for this and other variables are presented in Table 1.

Interest in computer science was constructed to gauge the degree of interest a subject had in computer science. The full list of statements used for this measure is also included in the appendix. As with the former scale, this scale was constructed by using a participant's mean score across all indicators.[2] A reliability analysis indicated that these indicators scaled together with very high reliability $(a=.930)$.

\section{Independent Variables}

The primary independent variables are related to gaming, including a scale measuring the diversity of gaming experiences and an indicator measuring the hours spent per week playing games. To test gaming diversity, the scale taps into participants' experiences with different genres of games and how often they play them. Although gaming genres have considerable overlap, the different genres of video games were broken down into 14 categories largely based around the standard industry breakdown of genres. Genres vary in terms of the type of game play, skills needed to complete the game, degree of interaction with other players (both 
online and offline), and the game's emphasis on narrative. For each genre, subjects were asked how often in the past six months they have played that type of game. The genres consisted of casual, arcade/retro, group entertainment, role playing style, simulation, action, adventure, strategy, racing, independent, shooter, massively multiplayer online (MMO), realistic sports, and fighting. For clarification, each genre listed two to four examples of well-known games. Subjects were also given two write-in options for games and genres not covered by the survey. Because nearly all responses actually did fit one of the existing categories, these answers were recoded into existing genres that best represented the write-ins.

For each genre, subjects were given a seven-point scale from 'never/have not heard of this genre' $(0)$ to 'daily or almost daily' (6). All the genres were then combined into a scale using the participant's mean score across all the indicators. This scale gives higher scores to players who play video games often, but also regularly plays different types of games. This means that subjects who responded that they play multiple genres will receive higher scores on average than those who play only one genre, regardless of how long they play that genre. This measure is particularly pertinent to studying attitudes toward computers, as different games require different skill sets of the player. First-person shooter style games, for example, generally require threedimensional spatial reasoning, but strategy games focus on other skills, such as resource management. The scale for this measure has high reliability $(a=.866)$.

The other variable used for gaming was hours spent playing video games per week. Participants were asked the question, 'how many hours a week do you spend playing video games of any kind?' Responses in whole hours were entered by the participants. This represents a more traditional measure for gaming involvement and genre experiences in favor of simply using the total amount of gameplay.

In addition to the gaming measures, a variety of demographic variables were also used as control variables. These include age first used a computer, parents' education level, family income, gender, current age, and race/ethnicity. Age first used a computer included responses of: 4-6 years old (0), 7-9 years old (1), 10-12 years old (2), 13-15 years old (3), 16-18 years old (4), and 19 years or later (5). Parents' education level was measured with separate questions for mother and father. Responses were coded as less than high school (0), high school (1), two-year college degree (2), four-year college degree (3), master's degree (4), and doctorate, including Ph.D., M.D., and J.D. (5). Family income was measured using a ten-point scale from less than $\$ 10,000$ to $\$ 91,000$ and above. Current age was measured with categories of: $18(0), 19$ (1), 20 (2), 21 (3), 22 (4), 23 (5), and 24 or older (6).

Table 1. Descriptive Statistics

\begin{tabular}{lrrrrr}
\hline & Median & Mean & SD & Min & Max \\
Confidence with Computers & 2.75 & 2.81 & 0.97 & 0.31 & 5.00 \\
Interest in Computer Science & 2.31 & 2.49 & 1.06 & 0.08 & 5.00 \\
Gaming Diversity Score & 1.50 & 1.55 & 1.04 & 0.00 & 4.79 \\
Gaming, Hours per Week & 5.00 & 8.85 & 10.65 & 0.00 & 70.00 \\
Age First Used Computers & 2.00 & 1.73 & 1.24 & 0.00 & 5.00 \\
Mother's Education & 2.00 & 2.30 & 1.21 & 0.00 & 5.00 \\
Father's Education & 2.00 & 2.27 & 1.32 & 0.00 & 5.00 \\
Family's Annual Income & 5.00 & 5.38 & 2.91 & 0.00 & 9.00 \\
Gender (Female) & --- & .49 & --- & 0.00 & 1.00 \\
Age & 2.00 & 2.43 & 1.89 & 0.00 & 6.00 \\
Race/Ethnicity (Black) & --- & .06 & --- & 0.00 & 1.00 \\
Race/Ethnicity (Hispanic) & --- & .06 & --- & 0.00 & 1.00 \\
Race/Ethnicity (Asian) & --- & .05 & --- & 0.00 & 1.00 \\
Race/Ethnicity (Other Non-White) & --- & .05 & --- & 0.00 & 1.00 \\
& & & & & \\
\hline
\end{tabular}

\section{Analyses}

The dependent variables are both normally distributed with skewness and kurtosis levels within normal tolerance ranges, so ordinary least squares (OLS) regression will be used. Models will be estimated first for confidence with computers and then for interest in computer science. For each of these dependent variables, one model will be estimated with just the gaming predictors, one with just the control variables, and one with all variables included. This will allow for a comparison of how the effect of gaming compares to the effect from the other variables combined, providing greater insight in the final model in regards to how much the control variables affect the gaming variables and vice versa. 
completing the survey part-way through. These participants were deleted listwise as they did not provide enough data to be included. Second, an additional 14 participants were similarly removed because they did not provide sufficient responses (i.e., answered fewer than half of the indicators) to reliably determine a mean score for the dependent variables. Finally, multiple imputation was used for the remaining cases missing data. Most cases $(80 \%)$ were complete, and very few (5\%) were missing on more than one indicator. Even the most commonly missing variable (family annual income) was still present for the vast majority of cases (92\%).

\section{Results}

The results for the models predicting confidence with computers are presented in Table 2. Model 1a estimates the effects of gaming involvement on confidence with computers. Both measures of gaming are statistically significant as predictors of confidence, with those who play a larger variety of game genres and those who play more often having higher levels of confidence with computers. The standardized effect for gaming diversity is far stronger than that of hours spent playing (. $441 \mathrm{vs.} .072$, respectively). Model $1 \mathrm{~b}$ estimates the effects of other variables on confidence. The age at which someone first used a computer is statistically significant, with those who started using a computer at a later age having lower confidence, all else being equal. Additionally, women also reported significantly lower levels of confidence with computers. Older individuals, on the other hand, had significantly more confidence than younger individuals, though the effect size was quite weak.

Table 2. OLS Regression Results Predicting Confidence with Computers

\begin{tabular}{llllllllll}
\hline & \multicolumn{2}{c}{ Model 1a } & \multicolumn{3}{c}{ Model 1b } & \multicolumn{3}{c}{ Model 1c } \\
\hline & $\mathrm{b}$ & $\mathrm{SE}$ & $\beta$ & $\mathrm{b}$ & $\mathrm{SE}$ & $\beta$ & $\mathrm{b}$ & $\mathrm{SE}$ & $\beta$ \\
\hline Gaming Diversity Score & .413 & .034 & $.441^{* *}$ & & & & .327 & .036 & $.350^{* *}$ \\
Gaming, Hours per Week & .006 & .003 & $.072^{*}$ & & & & .003 & .003 & .033 \\
Age First Used Computers & & & & -.206 & .023 & $-.263^{* *}$ & -.162 & .022 & $-.207^{* *}$ \\
Mother's Education & & & & -.051 & .026 & -.063 & -.055 & .025 & $-.068^{*}$ \\
Father's Education & & & & .005 & .024 & .007 & .014 & .023 & .019 \\
Family's Annual Income & & & & -.006 & .010 & -.017 & .002 & .010 & .007 \\
Gender (Female) & & & & -.738 & .055 & $-.379^{* *}$ & -.362 & .060 & $-.186^{* *}$ \\
Age & & & & .041 & .015 & $.080^{* *}$ & .061 & .014 & $.119^{* *}$ \\
Race/Ethnicity (Black) & & & & -.068 & .114 & -.017 & -.031 & .107 & -.008 \\
Race/Ethnicity (Hispanic) & & & & -.076 & .113 & -.019 & -.093 & .106 & -.023 \\
Race/Ethnicity (Asian) & & & & .078 & .129 & .017 & .116 & .121 & .026 \\
Race/Ethnicity (Other Non-White) & & & & -.102 & .129 & -.022 & -.139 & .121 & -.031 \\
& & & & & & & & & \\
Intercept & 2.113 & .049 & & 3.571 & .106 & & 2.674 & .125 & \\
\hline $\mathrm{R}^{2}$ & & & .242 & & & .224 & & & .320 \\
\hline
\end{tabular}

$* \mathrm{p}<.05, * \mathrm{p}<.01$

Model 1c combines the previous two models and estimates the effects of all predictor variables on confidence with computers. The conclusions reached here are largely similar to those of the preceding models. Notably, hours spent playing games has lost about half its effect size ( $\beta=.072$ to $\beta=.033$ ) and dropped to nonsignificant. In comparison, gaming diversity, though weaker $(\beta=.441$ to $\beta=.350)$, is still significant and has the strongest effect in this model. Also different from Model $1 \mathrm{~b}$ is the significance of the effect from mother's education, which has gained significance and indicates that those who have mothers with greater education levels have significantly less confidence with computers, running counter to what would be expected.3] Another noteworthy change is that the effect size from gender has declined substantially after controlling for the gaming variables ( $\beta=-.379$ to $\beta=-.186$ ). Comparing the three models, especially the coefficients of determination, it is clear that the impact of gaming is a strong effect. In addition to gaming diversity being the strongest effect, including the gaming measures increased the predictive power of the model by roughly $43 \%\left(\mathrm{R}^{2}=.320\right.$ compared to .224).

The results for the models predicting interest in computer science are presented in Table 3. Model 2a estimates the effects of gaming involvement on interest in computer science. Both measures of gaming are statistically significant as predictors of interest, with those who play a larger variety of game genres and those who play more often having higher levels of interest in computer science. As found when examining confidence with computers, the standardized effect for gaming diversity is far stronger than that of hours spent playing ( $\beta=.462$ verses $\beta=.079$ ). The results for Model $2 b$ are somewhat similar to the results from Model $1 b$. Here, those who starting using computers at a later age, and women, have significantly lower interest in computers, while those who are older have significantly higher interest. 
Table 3. OLS Regression Results Predicting Interest in Computer Science

\begin{tabular}{llcccccccc}
\hline & \multicolumn{3}{c}{ Model 2a } & \multicolumn{3}{c}{ Model 2b } & \multicolumn{3}{c}{ Model 2c } \\
\hline & $\mathrm{b}$ & $\mathrm{SE}$ & $\beta$ & $\mathrm{b}$ & $\mathrm{SE}$ & $\beta$ & $\mathrm{b}$ & $\mathrm{SE}$ & $\beta$ \\
\hline Gaming Diversity Score & .473 & .036 & $.462^{* *}$ & & & & .363 & .038 & $.355^{* *}$ \\
Gaming, Hours per Week & .008 & .003 & $.079^{*}$ & & & & .004 & .003 & .044 \\
Age First Used Computers & & & & -.184 & .024 & $-.215^{* *}$ & -.134 & .023 & $-.157^{* *}$ \\
Mother's Education & & & & -.048 & .028 & -.055 & -.052 & .026 & $-.059^{*}$ \\
Father's Education & & & & .027 & .026 & .033 & .037 & .024 & .045 \\
Family's Annual Income & & & & -.014 & .011 & -.038 & -.005 & .010 & -.012 \\
Gender (Female) & & & & -.916 & .059 & $-.431^{* *}$ & -.491 & .064 & $-.231^{* *}$ \\
Age & & & & .052 & .016 & $.093^{* *}$ & .075 & .015 & $.133^{* *}$ \\
Race/Ethnicity (Black) & & & & -.007 & .122 & -.002 & .036 & .114 & .008 \\
Race/Ethnicity (Hispanic) & & & & -.018 & .122 & -.004 & -.036 & .114 & -.008 \\
Race/Ethnicity (Asian) & & & & .244 & .138 & .049 & .290 & .129 & $.058^{*}$ \\
Race/Ethnicity (Other Non-White) & & & & .019 & .139 & .004 & -.021 & .129 & -.004 \\
& & & & & & & & & \\
Intercept & 1.691 & .052 & & 3.242 & .114 & & 2.229 & .133 & \\
\hline $\mathrm{R}^{2}$ & & & .268 & & & .249 & & & .351 \\
\hline
\end{tabular}

${ }^{*} \mathrm{p}<.05,{ }^{* *} \mathrm{p}<.01$

The final model, Model 2c, combines the previous two to include all the predictors in the same regression. As happened with confidence, the effect from hours spent playing games has dropped to nonsignificant once controlling for other factors and become substantially weaker ( $\beta=.079$ to $\beta=.044)$, and the unexpected negative effect from mother's education has slightly increased to the point of significance. Also newly significant is the effect from race, which indicates that Asian participants had significantly more interest in computer science. The effect from gender, though still significant, has shown a noticeable drop in effect size ( $\beta=-$ .431 to $\beta=-.231$ ). A comparison of the three models yields similar conclusions to those drawn from the confidence models. Specifically, gaming diversity was consistently the most powerful predictor ( $\beta=.355$ in the final model), and the gaming variables improved the model over a control-only model by $41 \%\left(R^{2}=.351\right.$ compared to .249$)$.

\section{Discussion}

The findings discussed here speak to the importance of leisure activities as a means of developing confidence and interest in related areas of one's life. This is particularly important for leisure activities associated with younger individuals as they form identities that will follow them as they begin making choices about their professional lives. Despite this, we often ignore the importance of leisure activities as influences of these decisions. Moreover, video games are often portrayed as a negative factor and seen as counter to professional life. Although each year video games become more accepted within mainstream culture, the public consciousness regarding video games still seems centered on Western FPS games, most often just referred to as 'violent video games.' This is problematic for two reasons: first, it ignores potentially positive outcomes from gameplay and, second, it ignores a variety of other genres that make up the large and diverse set of experiences to which gamers might be exposed. The present study sought to overcome these limitations by examining prosocial outcomes - confidence with computers and interest in computer science - as a possible outcome of exposure to more diverse gaming experiences.

The findings of the study indicate that there is a clear connection between playing video games and confidence with computers, as well as an interest in computer science. This suggests that playing video games may lead to a wider skill set based on the confidence with computers gained by consistently interacting with technology. Because video games give players a chance to interact with technology in an entertaining environment they are given an opportunity to learn tangentially about the logic involved in computer programs in a way that is stimulating and safe. The greater the variety of games an individual plays, the more chances they have to see the limits and advances of technology in action and interact with them in a way that is difficult to achieve outside of gaming. Moreover, the experimentation afforded to individuals through gaming can then lead to an interest in computer science as more avid players can be inspired to be involved in the creation of games. This echoes the findings of other research which demonstrated the development of specific skills through gaming (Cherney 2008; De Lisi \& Wolford 2002; Feng et al. 2007; Green \& Bavelier 2007; Lisk et al. 2012; Mayo 2009). These implications are particularly important in relation to the findings of DiSalvo et al. (2014), which noted the influence of game testing in encouraging an interest in computer science. Although a job play-testing games is an ideal circumstance for quickly introducing students to both video games and the basics of video game creation, these jobs are also limited. The current study suggests that being exposed to a variety of different game genres alone may increase the interest in computer science among students as a more practical alternative. This also 
means that the stigma surrounding video games as being an unproductive activity for young adults may be very harmful to their ability to establish and interest in computer science.

The results of this study also highlight how playing from multiple genres of games is an important aspect of gameplay that must be considered by future research studying video games. Specifically, those who played a larger variety of video game genres were more likely to feel confident in their technical abilities and to have an interest in computers, whereas the measure of gameplay through hours alone was not significant. According to the Electronic Software Association (ESA), shooters only make up $20.0 \%$ of console genres sold and make up only $7.1 \%$ of computer games sold in 2014 (ESA 2014). Although FPS games do not make up the entirety of violent gaming experiences, this does illustrate that the genres of video games are much more diverse than is often presented. When the research literature focuses predominately on one type of game (e.g., Carnagey et al. 2007; DeCamp 2015; Ferguson et al. 2014), it both ignores a large potential pool for research and gives the impression that FPS games represent video games as a whole. This is problematic both in that it is an artificial limit on studies, and also that FPS tend to be predominately western games and privilege male narratives. By excluding the benefits of the large number of games with non-masculine narratives, it continues to perpetuate the idea of gaming as a male dominated environment.

It should be acknowledged that this study is not without some limitations. Primarily, time-order cannot be fully established with the data used. Although the primary measure for gaming was based on the past six months and the measures for confidence and interest were present-time, one expects the relationship to expand far beyond that time frame and extend into childhood. Given that video games are marketed heavily towards children and teens, it could be expected that an interest in video games would be cultivated before an interest in computer science in the majority of individuals. Future research with youth participants and/or longitudinal data would be ideal for further investigating this possibility.

The data and analyses in this research provide strong empirical support of a relationship between playing video games and confidence with computers and interest in computer science. These findings highlight the importance of issues of inclusiveness with respect to video games, particularly for women and racial/ethnic minorities. Although additional studies are needed in order to conclude that this effect is occurring more broadly, these findings suggest that the inclusion of more diversity within gaming content and culture may encourage groups who have been largely been excluded from computer culture, particularly with regard to computer science, such as women. Overlooking the cultural significance of video games and their relationship to a growing technological culture has made it difficult to assess just how much impact exclusion may have for groups not well represented as players or within game content. More research and attention to these kinds of effects would be beneficial, to say the least, as we begin to take video games more seriously as a new and growing medium.

\section{Appendix: Statements used for dependent variable scales}

\section{Confidence with Computers \\ I often help my parents with computers}

I have considered studying computer science

I use computers for entertainment regularly

I often help other people with computer problems

I believe I am capable of learning computer programming (or a new programming language I am not familiar with)

I would rather fix computer problems I have encountered before by myself before asking someone do it for me

I can navigate menus for new operating systems or programs with ease

I enjoy showing someone how to do something new, more efficiently, or interesting on a computer

I believe it's worth my time to just play around on computers

I feel good when I've solved a problem with a computer

I am surprised when friends ask me for computer advice [reverse-coded]

I feel confident I can learn how to use new computer programs easily

I feel comfortable using computer programs with which I am not already familiar

I could install a hard drive into a computer if I needed to (or other hardware I have not installed in the past)

If I have a computer problem I haven't seen before I always try to fix it on my own before asking for help 
I avoid computers when I don't need to use them [reverse-coded]

Interest in Computer Science

I have considered studying computer science

I have considered pursuing a major or minor in computer science; I am interested in building my own desktop

A computer science degree would offer a lot of job security

I don't really care how computers work [reverse-coded]

A computer science degree is a very useful degree to have

I often help other people with computer problems

I believe it's worth my time to just play around on computers

I feel good when I've solved a problem with a computer

Taking computers apart can be fun

I could install a hard drive into a computer if I needed to (or other hardware you have not installed in the past)

If I have a computer problem I haven't seen before I always try to fix it on my own before asking for help

It is important to keep up with trends in technology

\section{Notes}

Reminder emails were only sent to those who neither participated nor opted out via a one-click unsubscribe link. Although there is no way to determine whether students with an interest in technology were more likely to respond to a web-survey in this population, the data indicate that $6 \%$ of respondents were computer science majors. This is higher than the school's proportion of computer science majors, yet it is not remarkably different, suggesting only a slight bias. Moreover, the age of this sample and population would suggest a high comfort level with technology (including mobile devices, which could be used to access the survey) and American college students have regular access to technology, making the survey easily accessible for all students asked to participate.

A factor analysis suggests that the variables for confidence and interest scales combine for their respective composite measures best with a single factor solution. For confidence, the first factor includes $44 \%$ of variance for the 16 indicators, compared to only $7 \%$ for a second factor. For interest, this comparison is $47 \%$ to $11 \%$. In both cases, it was decided to use only one factor given these results. All variables loaded well on the first factor, though the reverse-coded ones tended to form separate factors as well, likely as a byproduct of the methodological difference in response patterns. For simplicity, we chose to use the mean score across indicators rather than using factor loadings for the analyses presented here. Identical analyses using scores through factor extraction resulted in similar results leading to identical conclusions.

This effect is only found when the other predictors are included in the model. In bivariate regression models predicting confidence and interest (not shown, the effect is not significant and substantially weaker ( $\beta=$ .012 and $-.004, p=.71$ and .91 ). This does not invalidate the findings of the multivariate regressions, but does indicate that such results need to be interpreted carefully in the full context (i.e., that the effect is only present as found when the other variables are being controlled for).

\section{References}

ADACHI, P J, and WILLOUGHBY, T (2011) The effect of video game competition and violence on aggressive behavior: Which characteristic has the greatest influence?. Psychology of Violence, 1, p. 259-274. [doi:10.1037/a0024908]

ADACHI, PJC and WILLOUGHBY, T (2013) More than just fun and games: the longitudinal relationships between strategic video games, self-reported problem solving skills, and academic grades. Journal of Youth and Adolescence, 42, p. 1041-1052. [doi:10.1007/s10964-013-9913-9]

ANDERSON, C A, SAKAMOTO A, GENTILE, D A, IHORI, N, SHIBUYA, A, YUKAWA, S, NAITO, M, and KOBAYASHI, K (2008) Longitudinal effects of violent video games on aggression in Japan and the 
BARTHOLOW, BD, SESTIR, M A, and DAVIS, EB (2005) Correlates and consequences of exposure to video game violence: hostile personality, empathy, and aggressive behavior. Personality and Social Psychology Bulletin, 31, p. 1573-1586. [doi:10.1177/0146167205277205]

BLACKER, KJ, CURBY, KM, KLOBUSICKY, E, and CHEIN, JM (2014) Effects of action video game training on visual working memory. Journal of Experimental Psychology40(5), p. 1992-2004. [doi:10.1037/a0037556]

BUSHMAN, B J, and POLLARD-SACKS, D (2014) Supreme Court decision on violent video games was based on the First Amendment, not scientific evidence. American Psychologist, 69, p. 306-307. [doi:10.1037/a0035509]

CARNAGEY, N L, ANDERSON, C A and BUSHMAN, B J (2007) The effect of video game violence on physiological desensitization to real-life violence. Journal of Experimental Social Psychology, 43, p. 489496. [doi:10.1016/j.jesp.2006.05.003]

CREASEY, GL and MYERS, BJ (1986) Video games and children: effects on leisure activities, schoolwork, and peer involvement. Merrill-Palmer Quarterly, 32(3), p. 251-262.

CHERNEY, I (2008) Mom, let me play more computer games: they improve my mental rotational skills.Sex Roles, 59, p. 776-789. [doi:10.1007/s11199-008-9498-z]

DE LISI, R, and WOLFORD, J (2002) Improving children's mental rotation accuracy with computer game playing. The Journal of Genetic Psychology, 163(3), p. 272-282. [doi:10.1080/00221320209598683]

DECAMP, W. (2015). Impersonal agencies of communication: Comparing the effects of video games and other risk factors on violence. Psychology of Popular Media Culture, 4, p. 296-304. doi: 10.1037/ppm0000037 [doi:10.1037/ppm0000037]

DISALVO, B, GUZDIAL, M, BURKMAN, A, and MCKLIN, T (2014) Saving face while geeking out: video game testing as justification for learning computer science. The Journal of the Learning Sciences, 23, p. 272315. [doi:10.1080/10508406.2014.893434]

DONOHUE, SE, WOLDORFF, MG, and MITROFF, SR (2010) Video game players show more precise multisensory temporal processing abilities. Attention, Perception, and Psychophysics 72(4), p. 1120 1129. [doi:10.3758/APP.72.4.1120]

ENTERTAINMENT SOFTWARE ASSOCIATION (2014) Essential facts about the computer and video game industry Retrieved from http://www.theesa.com/wp-content/uploads/2014/10/ESA_EF_2014.pdf.

FENG, J, SPENCE, I, and PRATT, J (2007) Playing an action video game reduces gender differences in spatial cognition. Psychological Science, 18(10), p. 850-855. [doi:10.1111/j.1467-9280.2007.01990.x]

FERGUSON, C J, OLSON, C K, KUTNER, L A, and WARNER, D E (2014) Violent video games, catharsisseeking, bullying and delinquency: A multivariate analysis of effects. Crime and Delinquency, 60(5), p. 764-784. [doi:10.1177/0011128710362201]

FERGUSON, CJ and RUEDA, SM (2010) The Hitman study: Violent video game exposure effects on aggressive behavior, hostile feelings, and depression. European Psychologist, 15(2), p. 99-108. [doi:10.1027/10169040/a000010]

FERGUSON, C J, RUEDA, S, CRUZ, A, FERGUSON, D, FRITZ, S, and SMITH, S M (2008) Violent video games and aggression: Causal relationship or byproduct of family violence and intrinsic violence motivation?. Criminal Justice and Behavior, 35, p. 311-332. [doi:10.1177/0093854807311719]

GAMIRE, E. and PEARSON, G. eds. (2006) Tech Tally: Approaches to Assessing Technological Literacy. Washington, US: National Academy Press.

GEE, J P (2003) What video games have to teach us about learning and literacyNew York, NY: Palgrave Macmillan

GENTILE, D A, CHOO, H, LIAU, A, SIM, T, LI, D, FUNG, D, and KHOO, A (2011) Pathological video game use among youths: a two-year longitudinal study. Pediatrics, 127(2), p. e319-e329. [doi:10.1542/peds.20101353]

GILL, D (2009) Usefulness of video game experience for students learning and creating digital 3-d.Visual Arts Research, 35(2): p. 109-121.

GREEN, S, and BAVELIER, D (2006) Effect of action video games on spatial distribution of visuospatial attention. Journal of Experimental Psychology: Human Perception and Performance, 32(6), p. 1465-1478. [doi:10.1037/0096-1523.32.6.1465]

GUNTER, W and KEVIN D (2012) Causal or spurious: Using propensity score matching to detangle the relationship between violent video games and violent behavior. Computers in Human Behavior, 28, p. 1348-1355. [doi:10.1016/j.chb.2012.02.020] 
HOFFMAN, A J (2014) Violent media games and aggression-is it really time for a mea culpa?American Psychologist, 69, p. 305-306. [doi:10.1037/a0035289]

JOHNSON, S (2005) Everything bad is good for you. New York, NY: Riverhead Books.

JOLLY, K (2008) Video games to reading: Reaching out to reluctant readers. The English Journal, 97(4): p. 8886.

KUNTER, L, and OLSON, CK (2008) Grand Theft Childhood: The Surprising Truth about Violent Video Games and What Parents Can Do. New York, NY: Simon and Schuster.

LISK, T C, KAPLANCALI, U T, and RIGGIO, R E (2012) Leadership in multiplayer online gaming environments. Simulation Gaming, 43(1), p. 133-149. [doi:10.1177/1046878110391975]

MANNELL, R.C. (2007). Leisure, health and well-being. World Leisure Journal 49(3), p. 114-128. [doi:10.1080/04419057.2007.9674499]

MAYO, M J (2009) Video games: A route to large-scale STEM education?Science, 323, p. 79-82. [doi:10.1126/science.1166900]

MOE, A. (2014) Healing through movement: the benefits of belly dance for gendered victimization. Affilia: Journal of Women and Social Work, 29(3), p. 326-339. [doi:10.1177/0886109913516454]

PÖLLÄNEN, S. (2015) Elements of crafts that enhance well-being: textile craft makers' descriptions of their leisure activity. Journal of Leisure Research 47(1), p. 58-78.

ROSENBERG, R S, BAUGHMAN, S L, and BAILENSON, J N (2013) Virtual superheroes; using superpowers in virtual reality to encourage prosocial behavior. PLOS ONE, 8(1), e55003.

[doi:10.1371/journal.pone.0055003]

YOUNG, MF, SLOTA, S CUTTER, AB, JALETTE, G, MULLIN, G, LAI, B, SIMEONI, Z, TRAN, M, and YUKHYMENKO, M (2012) Our princess is in another castle: A review of trends in serious gaming for education. Review of educational research, 82(1), p. 61-89. 